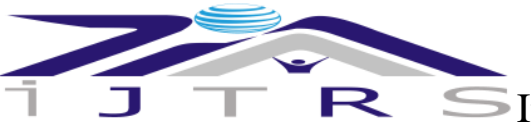

International Journal of Technical Research \& Science

\title{
MALIGNANT SKIN CANCER DETECTION USING CONVOLUTIONAL NEURAL NETWORKING
}

\author{
Rachakonda Hrithik Sagar ${ }^{1}$, Abhishek Bingi ${ }^{2}$, Aashray Pola ${ }^{3}$, Krishna Sai Raj Goud ${ }^{4}$, Tuiba Ashraf ${ }^{5}$,
} Subrata Sahana ${ }^{6}$

Email: hrithiksagar36@gmail.com ${ }^{1}$, bingi.abhishek@yahoo.com² ${ }^{2}$,aashraypolaa@gmail.com ${ }^{3}$, 2018006226.krishna@ ug.sharda.ac.in ${ }^{4}, 2018003670 . t u i b a @$ ug.sharda.ac.in ${ }^{5}{ }^{1}{ }^{\text {subrata.sahana@ @mail.com }}{ }^{6}$ Department of Computer Science Engineering, School of Engineering and Technology, Sharda University, Greater Noida, India

Department of Electronics and Computer Engineering, Sreenidhi Institute of Science and Technology, Ghatkesar, Hyderabad, India

Abstract- The incidence of skin cancer is increasing by epidemic proportions. According to WHO, Skin Cancer is the world's 6th most common cancer. It can be classified into Basal cell carcinoma, Squamous cell carcinoma and Melanoma among which Melanoma is more difficult to predict. By using this method, we can assist dermatologists to detect at an early stage as Computer Vision plays a vital role in diagnosis.

In this paper, to detect skin cancer we are using machine learning-based algorithms. Traditionally classification algorithms are Convolutional neural networking which Consists of initialization, adding a convolutional layer, summing pooling layer, summing flattening layer, summing a dense layer, then compiling Convolutional neural networks and fitting the CNN model to a dataset. We used machine learning model architecture to determine if the skin images of the patients are harmful or harmless via using machine learning libraries provided in python. We have chosen this approach to be more precise and specific in recognizing about cancer and ultimately declining the mortality rate caused by it

Keywords: Convolutional Neural Networking, Skin cancer, Malignant, Melanoma, Basal cell carcinoma, Squamous cell carcinoma.

\section{INTRODUCTION}

The main objectives of this research are:

$>$ To develop a prototype of a skin cancer-detecting system for Non-melanoma and melanoma skin cancer so that anyone who is reading this can develop their model.

$>$ To learn and Implement Convolutional neural networking model of deep learning to extract features from images that get trained and show output with great accuracies.

$>$ To help patients to prevent melanoma in early stages so that, that won't be leading to further skin problems.

Skin cancer is one of the diseases where it can put an individual's life at death door. At least 5.4 million people get diagnosed each year only in the US compared to lung cancer, breast cancer, colorectal cancer and prostate cancer [22]. The main parts which are detected for the cancer are the skin cells which do not grow at a normal rate on our body. A person falling prey for skin cancer may be in different forms but predominantly when exposed to Sunlight, its Ultraviolet radiation is the prime reason. The rate of being infected is inclining due to toxic substances present in our surroundings and a weakened immune system [6].

Skin cancer is one of the world's 5th common cancer according to the WHO. Skin cancer is divided into melanoma skin cancer and Non-Melanoma skin cancer. Basal cell carcinoma and squamous cell carcinoma comes under Non-Melanoma skin, compared to melanoma these are not dangerous if detected early. If not detected early twice as many people die as that of melanoma deaths. Last year 12000 people died of skin cancer out of which 9940 people died of melanoma and rest are Non-Melanoma skin cancer [7].

In the first category, we have basal and squamous cell carcinoma which are less likely to spread in different parts of the body. A gigantic number of more than 3 million people in America are being treated every year with this problem. 1 in 5 Americans is affected by skin cancer by the age of 70. Sunburns are also one of the risk factors for developing Melanoma to a large extent. The use of indoor tanning material is one of the pivotal cause for both melanoma and non-melanoma is calculated that a 10 per cent decrease in ozone levels will result in an additional 300,000 non-melanoma and 4,500 melanoma skin cancer cases. About $80 \%$ of non-melanoma skin cancers are basal cell carcinoma [8].

\subsection{Non-Melanoma Skin Cancer}

Non-Melanoma skin cancer can be found time and again and is curable. Every year 3 million-plus cases are reported for non-melanoma in the US and the number is rising every year. Organ transplant patients are more or less100 times plausible than the general public to develop squamous cell carcinoma. The rate of deaths gradually decreasing every year as we are detecting at early levels and getting advanced medical facilities [9].

DOI Number: https://doi.org/10.30780/IJTRS.V06.I05.001

pg. 1 


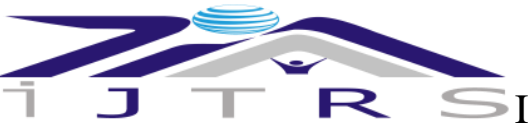

International Journal of Technical Research \& Science

Basal cell carcinoma, Squamous cell carcinoma and Merkel cell carcinoma are common types of Non-melanoma skin cancer. Basal cell is being diagnosed $80 \%$ more every year compared to rest. About 2000 people die every year with it. The latest statistical figures tell us that the number of deaths in America who are effected with squamous cell carcinoma is twice the number when compared to melanoma [10].

The risk factor for NMSC is:

$>$ About $95 \%$ of skin cancer patients can be treated if it is detected early for Non-Melanoma skin cancer.

$>$ Sun exposure: The Ultraviolet rays of the sun also damage skin, the more we expose to the sun the more we are likely to get exposed to skin cancer.

$>$ Use of tanning booths and sun lamps such as artificial tanning agents or ultraviolet rays if used before 30 years would lead to skin cancer too.

> Certain colours of skin, hair, and eyes: In Skin, people with blond hair or red hair and pale skin and green, grey or blue eyes tend to attract more towards Skin cancer.

$>$ People who had skin cancer before and whose family might have had it previously which would pass in DNA have increased risk of getting skin cancer. For example Actinic Keratosis.

$>$ Skin cancer depends on Age too, the older we get more we are prone to skin cancer.

$>$ Men's are more likely to get Non-Melanoma skin cancer than women.

> For people such as who had organ transplantation might have weak immune system those people are at higher risk and their skin cancer is more likely to be serious.

$>$ People who work in mining and are exposed to larger contents of arsenic tend to develop skin cancer.

$>$ People with a history of radiation treatment are at higher risk of skin cancer.

$>$ Skin cancer can develop in the areas where there might be scars, burns and inflamed skin.

$>$ Smoking also leads to skin cancer on the lips.

$>$ "Human papillomavirus infection" is also known as HPV infection. Some types of HPV can infect skin near the genital area and increase the risk of skin cancer around that place.

$>$ Risk of skin cancer is more at an early age for people who are with conditions such as basal cell nervous syndrome or xeroderma pigmentosum.

$>$ Medicines and some of the steroids that make the skin more likely to "sunburn" and that weaken the immune system and more probably to increase the risk of skin cancer. Such as voriconazole, vandetanib and vemurafenib. Medicines such as BRAF can increase risk of getting new NonMelanoma Skin Cancers.

$>$ If the anatomic distribution is more than $80 \%$ then skin cancer is more prone to the upper limb, head and neck.
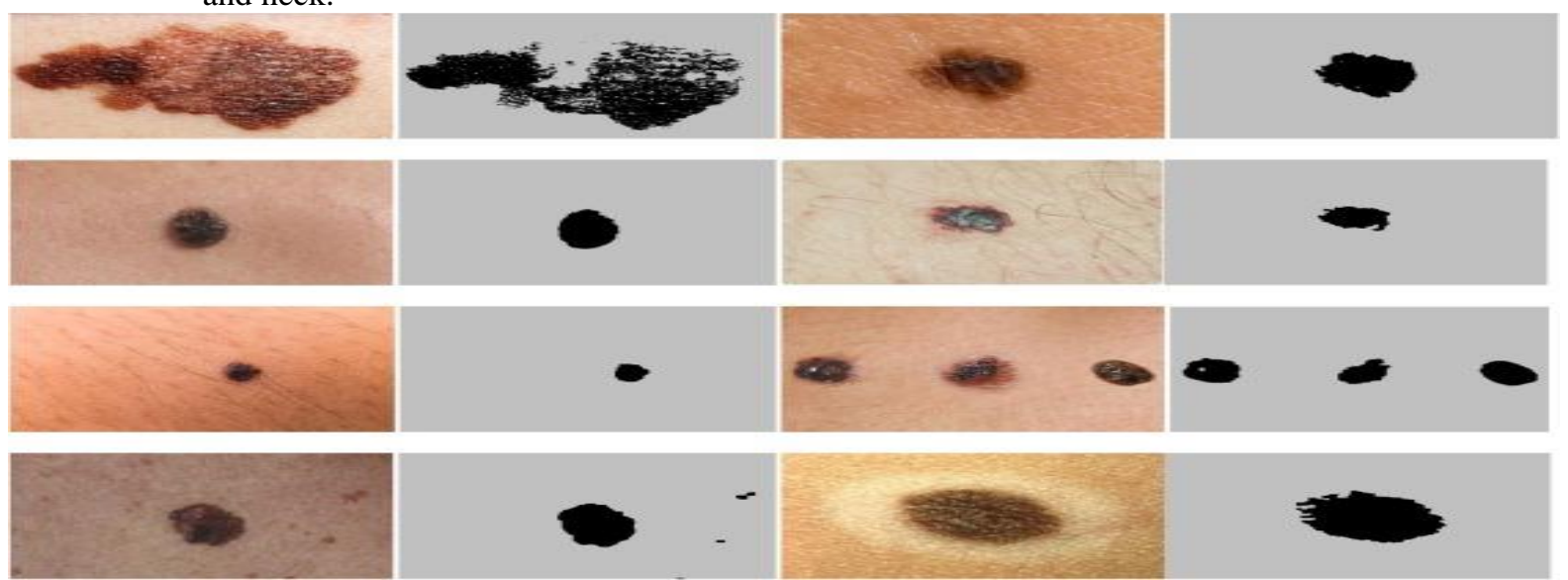

Fig. 1.1 Skin Cancerous Cells

$80 \%$ of all NMSC are basal cell carcinoma, which makes it a more common type. This is Low risk but highly disfigured by invading local tissue whereas other $20 \%$ is Squamous cell carcinoma and 2nd most cutaneous malignant and most directly correlated with chronic sun exposure, burn injuries scars, HPV virus [11].

Some of the symptoms of Non-Melanoma Skin cancer are:

$>$ Sore that doesn't heal doesn't come back after healing.

$>$ Yellow or pale white areas that look like scars.

$>$ Scaly and raised red patches.

$>$ Shiny, smooth and small pearly white or pink or red lumps.

$>$ Growth with small blood vessels on the surface.

$>$ Bleeding sore.

$>$ Itchy sore. 


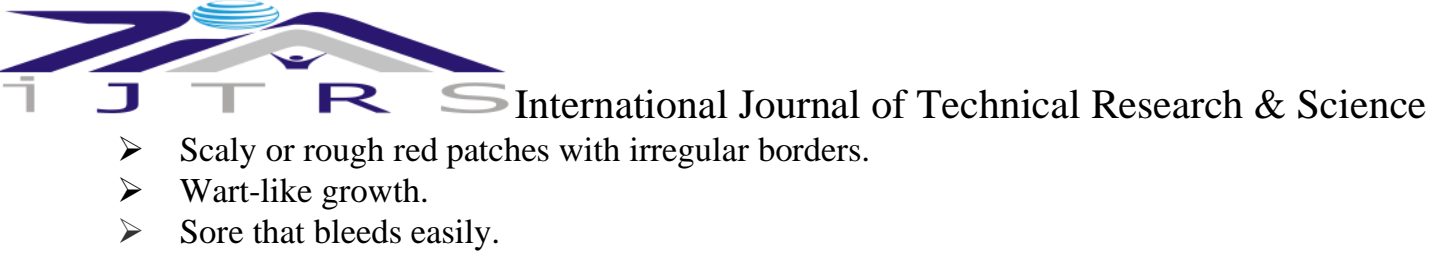

\subsection{Melanoma Skin Cancer [MSC]}

This is more threatening and prominent in men and women. As per net statistics of the year 2019, around 100,400 people are diagnosed with melanoma in the USA out of this 60,000 are men and 40,400 are women. This type is ranked 6th most occurring type among men and the 5th most occurring type of cancer among women. Unbelievably people that are diagnosed with skin cancer are remarkably older than 70 years. $90 \%$ per cent of white people are diagnosed with Merkel cell cancer. Women are twice less likely to be diagnosed than men [12]. The melanoma type has a drastic increase in the past few decades, although it depends on the age factor. If this is diagnosed at an early stage, it is cured by initial surgery. The number of people who are already living with skin cancer for about 5 years is identified by a "5-year survival rate". According to the reports people who got diagnosed in an initial stage their survival rate is 5 years is almost $90 \%$ but this depends on the thickness of the melanoma, the spread of melanoma to different parts of the body Lymph node involvement [13].

Increase of melanoma is over $700 \%$ per cent since the past 50 years. This is a lifetime risk. One person dies with melanoma each hour. Mostly happens to young women and the mortality rate is quite high. People who are detected with melanoma early indeed has 5 years of survival at least and 99\% curable [14].

Characteristics of melanoma are:

$>$ Cells of melanoma are asymmetrical when we draw a line from the centre, they don't collide nor be symmetrical.

$>$ Most of the cells have border irregularities and are recognized as the scalloped or notched edge.

$>$ Colour variability matters here, generally affected cells turn to brown, tan, black, and as progressing they turn to red, white and blue.

$>$ Moles are of higher diameter than normal moles and grow longer than common moles.

$>$ Evolution, the evolution of cells is higher than regular there exist more and more moles at one point of skin.

Places where melanoma can exist without our notice:

$>$ Melanoma can hide under fingernails or toenails, it knows as Acral-melanoma and is the rare form of melanoma. Most commonly present in people like Asian descent, black people and other people with dark skin.

- Melanoma can be in the nose, mouth, digestive tract, urinary tract or vagina this kind of melanoma is known as Mucosal Melanoma and these are very difficult to detect as they have similar conditions with other issues.

$>$ It can be in the eye; eye melanoma is also called ocular melanoma. Generally, they occur in the layer beneath the white of the eye this can cause eyesight, vision problem and can be diagnosed during an eye test.

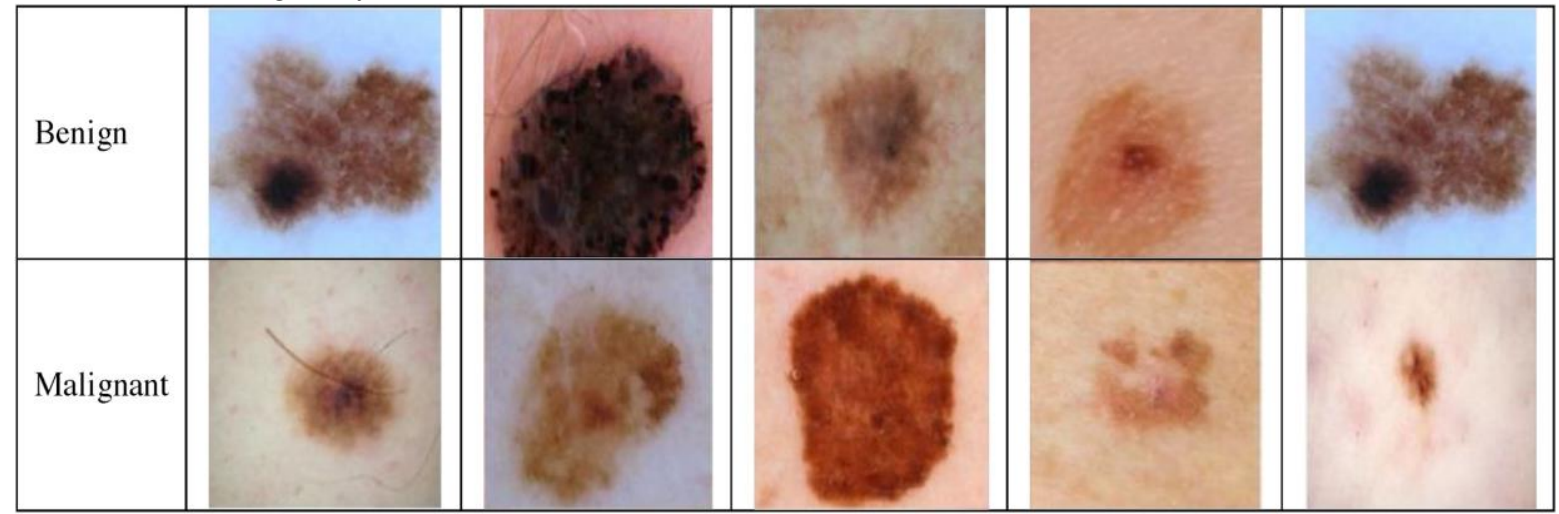

Fig. 1.2 Images of Benign vs Malignant Skin Cancer

Risk factors for MSC are:

$>$ Fair skin.

$>$ History of Sunburn.

$>$ Excessive Ultraviolet rays' exposure.

$>$ Generally, people who live close to the equator or who live at a higher temperature.

$>$ Having many moles or unusual moles.

$>$ Family history of Melanoma.

> Other inherited genetic conditions such as retinoblastoma, Li-Fraumeni syndrome, xeroderma pigmentosum.

DOI Number: https://doi.org/10.30780/IJTRS.V06.I05.001

pg. 3

Paper Id: IJTRS-V6-I4-001 www.ijtrs.com, www.ijtrs.org 


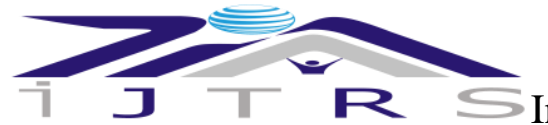

\section{SIGNIFICANCE OF THE SYSTEM}

The primary focus of this paper is, how Machine Learning Techniques are being implemented in its most archaic nature applied in detecting skin cancerous cells before scathingly dangerous effects on the human's lives. Its study on literature survey is written in section III, Ease of use is given in section IV, Section V describes Dataset that we have used in the algorithm and for references, Background work of the paper is described in section VI, Methodology is being elaborated in section VII, section VIII consists of the results that have been tested and verified by the model, section IX has the conclusions on the paper and its methodology.

\section{LITERATURE REVIEW}

Although there are many types of Neural Networking architectures after a thorough analysis of these architectures, Convolutional neural; networking works better in detecting Skin Cancer as it entirely deals with image processing and has a lot of support for image analysis such as it's able to help users extract features very distinctively, has high accuracy, it follows a hierarchical model which works on building an entire network and then provide a fully connected layer at the end where every neuron are connected and the outputs are processed. Most importantly convolution layers are built for images processing which helps in detecting features that can be missed in simply flattening images pixels values and is done automatically without any human supervision. It creates classes itself without the help of human otherwise which is a big task and CNN is computationally efficient. The link between adaptive filters and feed-forward neural networks are known as CNN. All these claims are being verified by trustable sources [1].

Most of the other models that are detecting Skin cancer are using Support vector Machines (SVM), Artificial Neural Networking (ANN), K-Means. Compared to these CNN was giving more better results as this is fully based on Image processing and build for this sole purpose [2]. Both SVM and CNN are Supervised machine learning algorithms where $\mathrm{CNN}$ is a parametric classifier which uses hyper parameters during the training phase and SVM is a non-parametric classifier which finds a linear line to separate classes and mainly used for classification and regression analysis whereas CNN is non-linear, this works well with visual image recognition and increases model complexity by adding more and more layers. On huge data, CNN works far better than SVM [3].

According to a study done by "Abien Fred M. Agarap" written in this paper "An Architecture Combining Convolutional Neural Network (CNN) and Support Vector Machine (SVM) for Image Classification" [4] has proven that Convolutional neural networking gives the accuracy of $99.23 \%$ while Support Vector Machine gives out the accuracy of $99.04 \%$ which gets CNN lead over SVM. He has performed a detailed analysis of both the techniques [5].

\section{EASE OF USE}

Generally, the goal of this paper is to achieve the maximum probability of predicting skin cancerous cells which makes patients check within the app instead of visiting hospitals and going through conventional methods. Hence, the final goal is to reach the maximum possibility and audience.

According to the survey we have done, we have tested our program on 20 people and trained over 2435 images and tested on 450 test data, this program was giving 8 out of 10 right outputs

The conventional way would be long and costs a certain amount of money for a patient whereas if they use this technique they will get to know whether they are suffering from skin cancer or not up to certain probability free of cost, hence without this model it'll be hard for most of the below middle-class people to afford hospitals as it costs around 7000 Rupees to 20000 Rupees per person for testing. Therefore it will be good to visit hospitals if we are confirmed that we are positive with skin cancer.

To access these users just need to run the "SSC_Main_2" file and put their skin image and run the file. Users can use this through their laptop at home or anywhere just with the help for laptop. Users don't need internet connectivity to access it.

Users need little knowledge of running the model even if they don't have they can learn it in simple steps.

\section{DATASET}

There are four sub-datasets for this dataset. The first dataset has basal cell carcinoma cancerous cells samples, then squamous cell carcinoma cells samples photos come melanoma cancerous cells samples then samples of skin cells which aren't cancerously known as benign these are samples of healthy cell tissues. This division helps us in getting an indication and for diving classes to train machine learning models. The dataset is taken from minimum 7 to 95 years old age range of people having cancerous cells on the skin.

\section{BACKGROUND}

Hence considering the loss and sacrifices due to Skin cancer and analyzing how to overcome the issue, the answer would be detecting it early. Most of the time for most of the people sin cancer might be in the form of normal Mole which we obviously think is mole without knowing the risk behind it we ignore which leads us to the bringer of death [15]. 


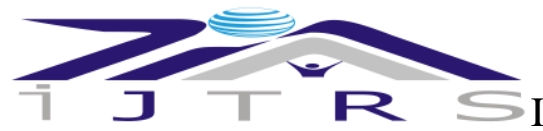

\section{International Journal of Technical Research \& Science}

The possible solution to this upcoming fatal incident would be by detecting it early, of course, there are many ways for this but most of the ways take a lot of time and needs to be travelled from one place to other such as Home clinic/hospital. There are other ways of self-checking for skin cancer to spot it early, and that's done in the following ways [16]:

Checking whole body thoroughly (including genital areas, toe/fingernails, back...etc.), new skin growth, irregular shapes, bumps, expanding of the skin or changing growth, rough red patches, bleeding skin, wart-like growing, odd-shaped moles/ changing in size of them/colour [17].

This is a long process and can't say accurately whether we are diagnosed with it or now. After considering this process it's good to detect skin cancer early so that the treatment would be given once it's late it'll be a problem. Thus, we need to do something which detects automated skin cancer cells of humans, so the diagnosis and treatment can be given before it grows dangerous and that is to detect skin cancer using machine learning and subdomain as image processing using neural networking this model is consistent, efficient and cost-effective as it was done purely from the open-source image processing techniques [18]. Although there are many ways of using image processing such as:

$>$ K-means algorithms.

$>$ Support Vector Machines algorithm.

$>$ Neural networking.

$>$ K-Nearest Neighbours algorithm.

$>$ Scale-Invariant feature transform algorithm.

$>$ Random sample consensus algorithm.

$>$ Cross-Validation algorithm.

\subsection{Neural Networks}

Let us understand the basic types of neural networks and their applications so that we will know which neural networking model to use of all the pre-present architectures:

\subsubsection{Feedforward Neural Networks:}

These are known as artificial neurons. Generally used for supervised learning. These are the simplest form of artificial neural networks and it has one direction input/output/data. These might have hidden layers to justify it just has front-propagation wave and no sign of backpropagation wave.

Summation of products of the inputs and weights are being calculated and fed to the output. If the threshold value is 0 then the output is considered to be above a particular value and neuron fires with the activated output of value 1. If the neuron does not fire then it usually emits -1 which means it's deactivated

Applications:

$$
\begin{aligned}
& >\text { Computer vision. } \\
& >\text { Speech recognition. } \\
& >\text { Pattern recognition. } \\
& >\text { Speech recognition. }
\end{aligned}
$$

\subsubsection{Radial basis function Neural Networks:}

Mentioned as RBF. This has layers in first features are being combined with radial basis function in the inner layer and output of it is taken into consideration. Which will be used while computing the same output in the next step which I s memory.

Applications:

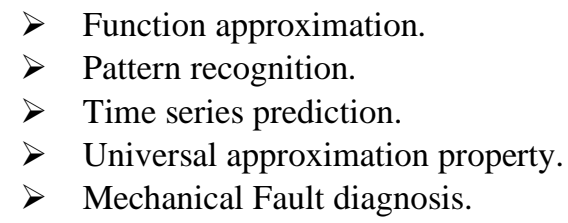

\subsubsection{Kohonen Self Organizing Neural Networks}

Type of ANN used to train unsupervised learning to get low dimensional, discrete representation of input space on training data called a MAP and it is known as "dimensionality reduction". Has either 1 or 2 dimensions.

This process has different kinds of parts, in the very first phase of self-organization, every neuron value is initialized with a small weight and the input vector. In $2^{\text {nd }}$ phase, here comes "Winning Neuron" which is closest to the neuron, and neuron connected to this neuron will move towards closest winning neuron and this distance is calculated using Euclidian distance and the neuron with less distance wins.

Applications [19]:

$>$ Pattern recognition.

$>$ Medical analysis to clustering data into different categories.

> Tool monitoring.

$>$ Static security assignment. 




\subsubsection{Recurrent Neural Network (RNN) - Long Short-Term Memory}

RNN works by saving the output of a layer, then feeding same back to the input to help in the prediction of the outcome of the layer.

In this $1^{\text {st }}$ layer is formed similar to feed-forward $\mathrm{NN}$ with the product of the features and sum of weights. The RNN starts once this is computed, i.e. one-time step to the next each neuron will remember some information it had in the previous step. This makes every neuron act like a memory cell in performing computations. For that process, we need to let RNN work on the forward propagation and store in memory what information it needs for later use. Here, if the predicted output is wrong we use the learning rate or error correction to make small changes so that it will gradually work towards making the right prediction during the backpropagation [20].

Applications of recurrent neural networks:

$>$ Text to speech conversion.

$>$ Text conversion of Human-like speech.

\subsubsection{Convolutional Neural Network (CNN)}

CNN's are very much similar to feed-forward neural networks, whose principles are having learnable weights and biases.

CNN is a Deep Learning algorithm which takes input in form of an image, assigns importance such as biases and learnable weights to various features/objects in the image and then can differentiate one from the other [18].

Applications:

$>$ Signal and image processing.

$>$ Computer vision.

$>$ Image processing.

$>$ Image classification.

$>$ Image analysis and recognition.

$>$ Facial recognition applications.

$>$ Social media.

$>$ Recommender systems.

$>$ Document digitization.

\subsubsection{Modular Neural Network:}

These have different kinds of networks collections which work independently and contribute outputs. Each neuron contributes a unique set of values of input compared to another kind of network-constructions and then performs later tasks. While accomplishing tasks these don't interact with each other networks [21].

Advantages of these are: Large computational process are breakdown into small thus decreasing complexities, which helps in reducing the number of connections hence negotiating neural network interactions with others which leads to computations speed fastening though this time depends on the involvement of neurons in computations and their number.

Applications of the modular neural network are:

$>$ Aircraft trajectory prediction.

$>$ Pattern recognition.

$>$ Grade estimations.

$>$ Biometrics.

\section{PROPOSED SYSTEM}

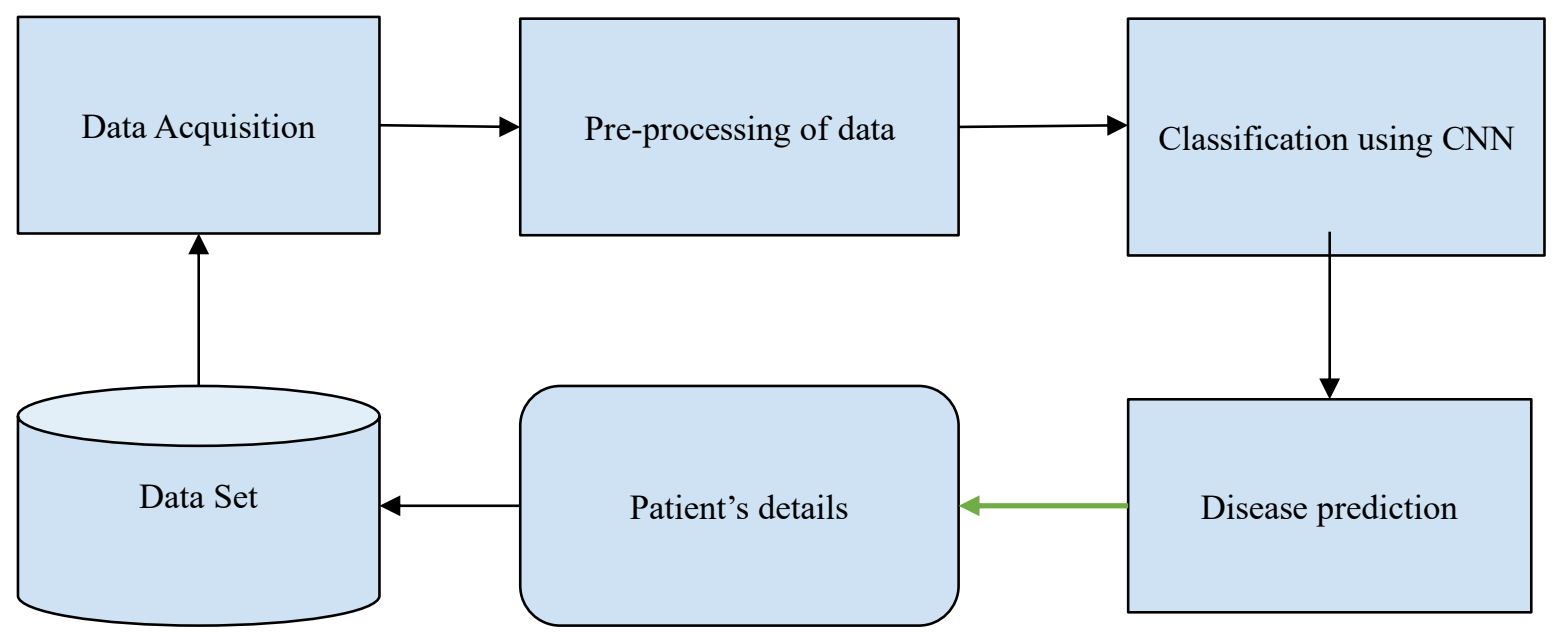

Fig. 7.1 Flow Chart Representation of CNN

DOI Number: https://doi.org/10.30780/IJTRS.V06.I05.001

pg. 6 www.ijtrs.com, www.ijtrs.org 


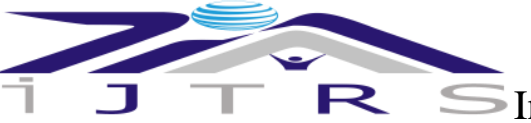

So let us see what happens with data in CNN in brief:

In the above flow chart Fig. 7.1, detailed explanation of titles:

\subsection{Data Acquisition}

This is the sampling real-world data then converting it to digital values which can be used by a computer to process them, Generally waveforms are converted into digital form, in our case skin image in the format of JPEG are converted into digital format and then used in the next step, pre-processing.

$>$ Pre-processing of data: let us understand this by taking reference of the dataset images of skin used in the paper, this data is going to get encoded, transformed, bought to s state where the algorithm can understand it easily and the features of images are interpreted by it. Whose features might be vectors, patterns, matric values, entities, edges, corners, Scale-invariant Feature Transform, Speeded up robust feature.

> Classification using CNN: Though there are many other effective ways to classify data, CNN does it well with images and is preferred rather than other algorithms, this work with high dimensional pictures, deep learning framework like TensorFlow, which helps to get greater outputs at fewer lines of codes.

$>$ Disease prediction: Prediction of the disease in this case Skin cancer, in this step the algorithm predicts if the input image is diagnosed with skin cancer of not.

$>$ Patient details: This is the step where details of the patient are known to the algorithm and outputs them along with the disease prediction.

$>$ Data set: Dataset is taken from various networks and some are taken by the survey that's been done and this dataset is processed to the algorithm for going through processes written before this step.

\subsection{History of Convolutional Neural Networking}

Neural networking was first proposed by Warren McCullough and Walter Pitts in 1944, who were students of the University of Chicago then moved into MIT in 1952.

Convolutional Neural Networks are generally known as "ConvNets" and are considered to be the backbone of many computer vision systems. Aim of ConvNets is to find the correlation between adjacent inputs found in the image. CNN consists of one or more convolutional layers which are being followed by a fully connected layer and whose design is based on the 2D input image's structure.

The first work on Neural networking was done by "Dr Kunihiko Fukushima" which was inspired by "Hubel and Wiesel's" work on complex and simple cells and proposed "NEOCOGNITRON" model.

Then the first-ever work on CNN was done in the 1990s by "Yann leCun et al", inspired by The Neocognitron, though it was invented in 1980s the first official paper was launched in 1998 citing word deep convolutional network about 6184 times. This paper was widely appreciated and regarded as one of the most influential publications in the field of technology.

Later Alex Krizhevsky developed AlexNet around 2012 and that achieved "state-of-the-art performance" in "ImageNet challenge" and dataset consisted of 1.2 Million images of high resolution.

As Human vision and biological neural networks, CNN also follow "Simple-to-Complex" hierarchical structure, just that CNN's are build using mathematical operations [1], [3], [12], [19].

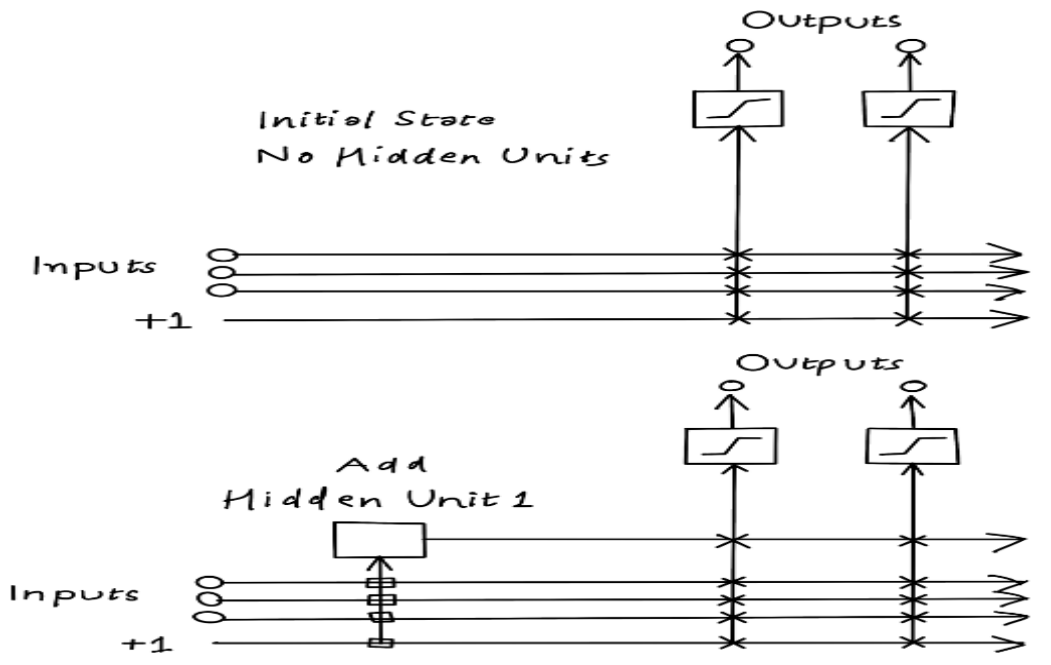

Fig. 7.2 Structure of a Neural Network

DOI Number: https://doi.org/10.30780/IJTRS.V06.I05.001

pg. 7

Paper Id: IJTRS-V6-I4-001

www.ijtrs.com, www.ijtrs.org 




Convolutional neural networking:

In a nutshell:

$>\mathrm{CNN}$ is a Deep Learning algorithm which takes input in form of the image, assigns learnable weights and biases to various features/objects in the image and then can differentiate one from the other.

> Similar to ANNs but more capable and advanced, CNNs are more complex around computation and pre-processing.

$>$ The input to the model is skin lesion image and then it's applied by conventional image processing techniques, it analyses those inputs to provide conclusion about the presence of cancerous cells.

> This Lesion Image analysing tools checks for the various Melanoma parameters Like Border, Asymmetry, Diameter, colour etc. by size, texture and shape analysis for image segmentation and feature stages.

In-depth:

Convolution neural networking is generally called as CNN/ ConvNets. In a nutshell, this is a Deep learning algorithm where the input would be an Image then assign importance to it based on various features and which will be able to differentiate one feature form other, which are known as weights and biases. CNN learns features, characteristics of the training images.

The most important feature of CNN's are time taken for "Pre-Processing" which would be much less when compared to other algorithms which perform classification. The architecture of ConvNets is similar to human brain neural networks pattern of connectivity and these are even inspired by visual cortex organization.

While coming to technical terms ConvNets train and test models each of the input images shall pass through series of convolution layers using filters known as kernels then through pooling layer then after that they get flattened then to fully connected layers and then applied with activation function known as Softmax whose probability functional values range between 0 and 1 .

The Softmax function is represented this way:

$$
P\left(y=j \mid \theta^{\wedge}(\mathrm{i})\right)=\left(e^{\theta^{\mathrm{i}}}\right) / \sum_{j=0}^{k} e^{\theta^{\mathrm{i}}}
$$

Where,

$$
\theta=\mathrm{W}_{0} \mathrm{X}_{0}+\mathrm{W}_{1} \mathrm{X}_{1}+\ldots \ldots+\mathrm{W}_{\mathrm{k}} \mathrm{X}_{\mathrm{k}}=\sum_{i=0}^{k} W_{i} X_{i}=W^{T} X
$$

Generally, photos are scaled to $(32 * 32 * 3)$ which corresponds to Width $(\mathrm{W})$, Height $(\mathrm{H})$ and Colour channels $(\mathrm{C})$ red, green, blue. Which means neurons are arranged in 3 dimensions (W, H, C). The final output layer would be the dimension of $(1 * 1 * 10)$ as by end image was pre-processed and flattened into a single vector arranged along with depth dimension along with class scores. Weights on the neuron are also calculated this way:

$$
\mathrm{Ex}: 32 * 32 * 3=3072
$$

3072 total weights are being processed in the neural network in one hidden layer of it. when image size increases the number of weights that needs to be processed increased which would take a lot more processing power, time and several more neurons and finally leads to overfitting.

Simply ConvNets are a sequence of layers and the most important layers to build neural networking architectures are Convolutional layer, pooling and Fully Connected layer. Then these layers are stacked upon each other to form fully connected neural networking architecture.

\subsection{Convolution Layer}

The Convolution layer is the core method of a Convolutional Neural Network. Its parameters consist of different learnable filters and every filter is small but gives a major feature that we need. ConvNets learns filters by which it activates when it detects the features such as colour, edge...etc. by this process, we will have a set of filters in each Convolution layer. Each one of them will create an activation layout by which we can detect output we need.

\subsection{Local Connectivity}

As Images are high dimensional inputs connecting neuron to neurons are impractical, instead, we connect each neuron to a local region of the volume of input. The special extent of that connectivity is defined by "Respective filed of neuron". The depth axis along with the field of the neuron is equal to the depth of input volume. The important part is to emphasize the asymmetry that occurs and how we treat that spatial dimensions such as height and width along with depth dimensions. The connections are always full with the depth of input volumes but are local inside space.

For example, let the size of input be [32×32x3] of volume, other examples might be RGB CIFAR-10 image. Let

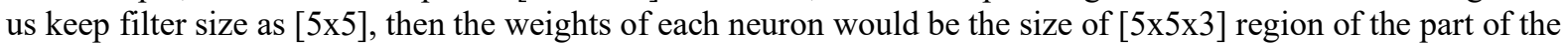
input volume, which would be making $75\{5 * 5 * 3=75\}$ weights then the bias of +1 would be added. Now change

DOI Number: https://doi.org/10.30780/IJTRS.V06.I05.001

pg. 8 
7 7 R International Journal of Technical Research \& Science

the notice in the depth axis along with the extent of the connectivity would turn out to be 3 , as this would be the input volume's depth.

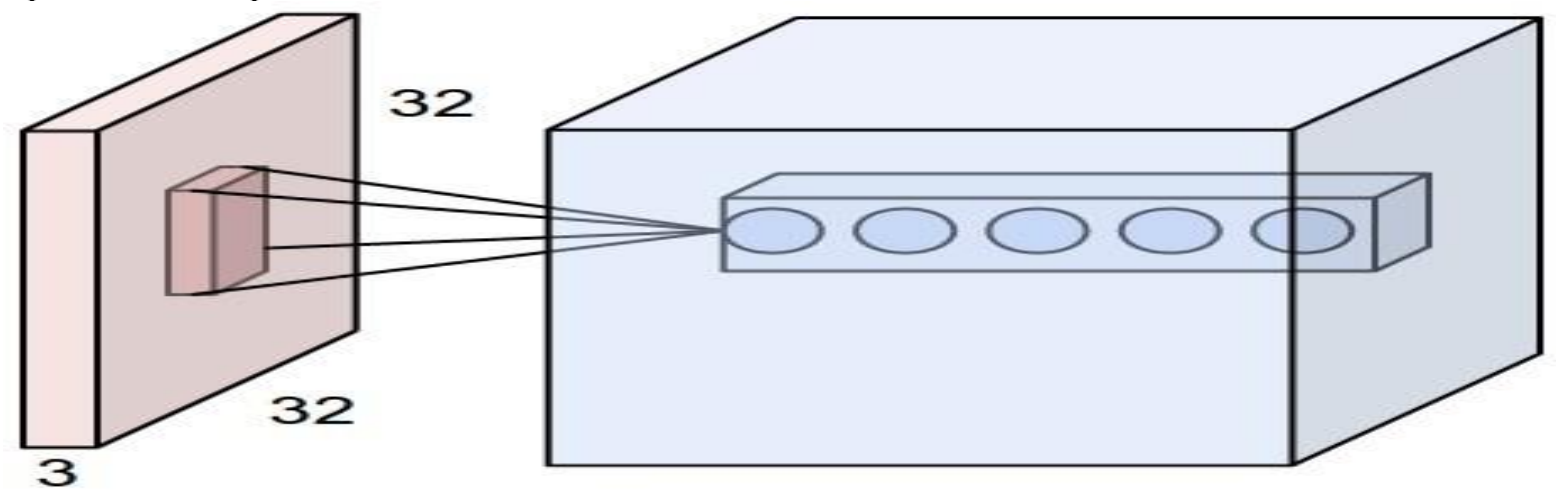

Fig. 7.3 Red, Example Input Volume
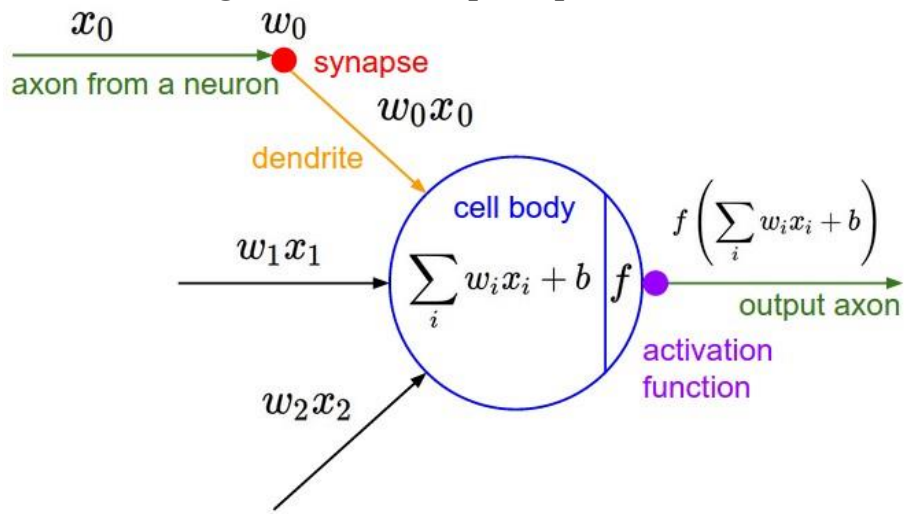

Fig. 7.4 Neurons that Remained Unchanged

\subsection{Spatial Arrangement}

Zero-padding, depth and Stride are the three main hyper-parameters which control the size of output volume. Refer above data for knowing more about them.

Table-7.1 Tabular representation of usage of depth, Stride and Zero-Padding

\begin{tabular}{|l|l|l|}
\hline \multicolumn{1}{|c|}{ DEPTH } & \multicolumn{1}{|c|}{ STRIDE } & \multicolumn{1}{c|}{ ZERO-PADDING } \\
\hline $\begin{array}{l}\text { The number of filters that are } \\
\text { being used. }\end{array}$ & Performs sliding of a filter. & $\begin{array}{l}\text { Zero-padding controls the } \\
\text { spatial size of outputs volumes. }\end{array}$ \\
\hline
\end{tabular}

Size of output volume is being calculated by the above formula:

Where,

$$
\text { OUTPUT VOLUME }=\frac{1((\mathrm{~W}-\mathrm{F})+2 \mathrm{P})}{\mathrm{S}}+1+\frac{((\mathrm{W}-\mathrm{F})+2 \mathrm{P})}{\mathrm{S}}
$$

$>$ Input volume size $=\mathrm{W}$

$>$ Receptive field size $=\mathrm{F}$

$>$ Stride $=\mathrm{S}$

$>$ Zero padding $=\mathrm{P}$
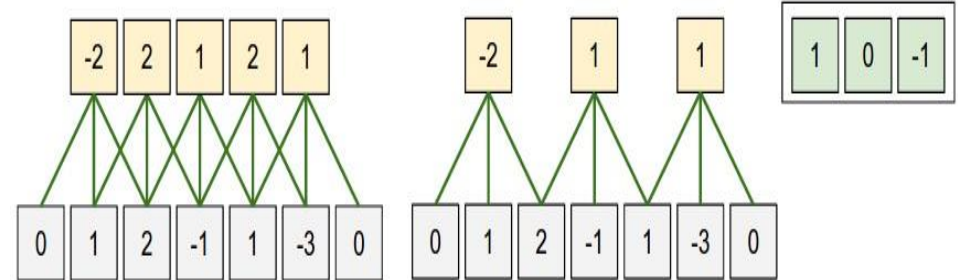

Fig. 7.5 Neurons using stride for clear Understanding

\subsection{Parameter Sharing}

Controlling the number of parameters is known as parameter sharing. Let's see a real-world example here:

We see that there are $40 * 40 * 85=136,000$ neurons in the very first convent layer and each neuron weight $16 * 16 * 3$ $=768$ (weights) and bias of value usually 1 together, they add up to $136,000 * 763=104,448,000$ parameters in the very 1 st layer itself and the number is very high that can be reduced by making depth slice which is the only valid 


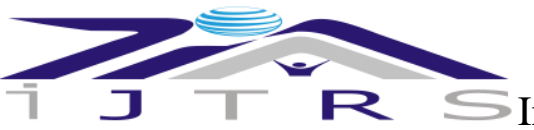

assumption. If every neuron in a single depth uses the same weight vectors and those are passed to the previous layer i.e. convolution layer, and each depth slice is computed neuron weights convolution along with input volume. Thus, that layer was given name as a convolution layer.

Hence, it's commonly referred to as "Filters/Kernels" (Sets of weights) which are convoluted along with inputs. Parameter sharing assumptions sometimes might not make any sense. In such types of cases it is common to relax parameter sharing scheme this is called a locally connected layer.

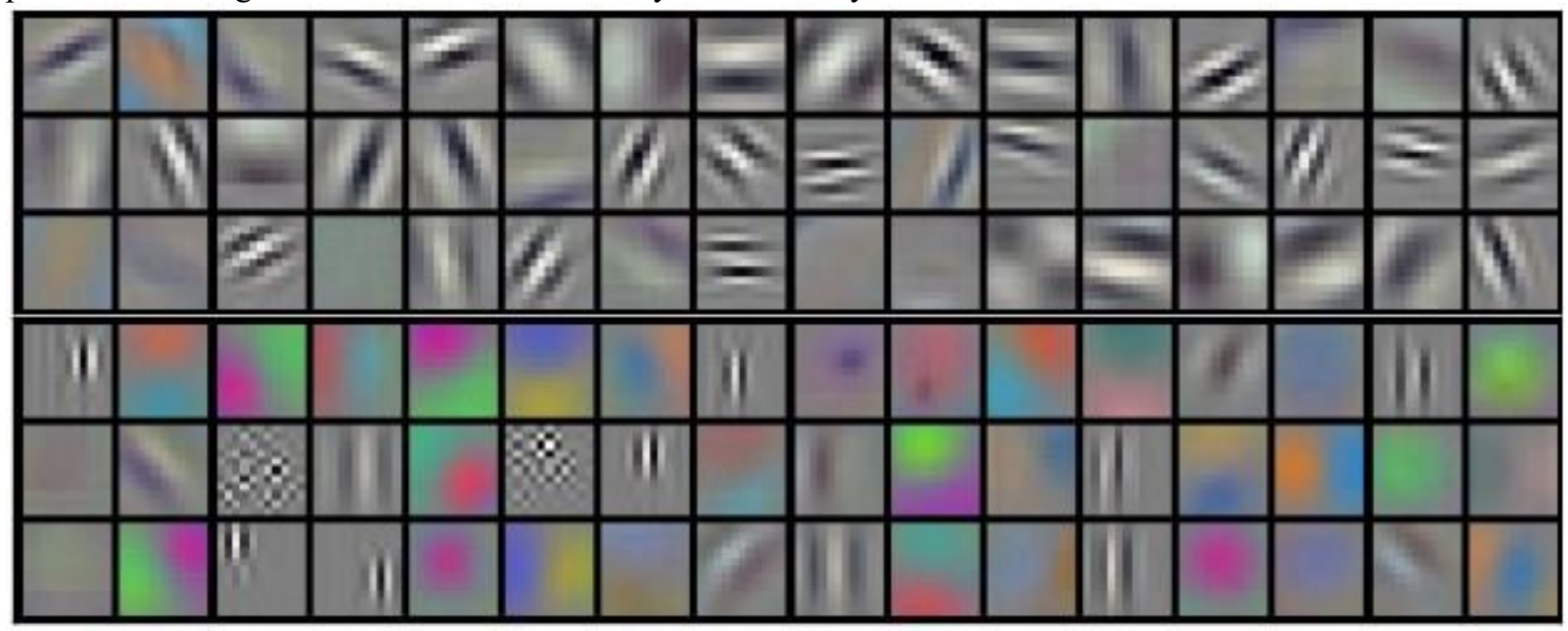

Fig. 7.6 Filters learnt by Krizhevsky et al in his paper, size $=55 * 55$ in 1 Depth Slice

\subsection{Pooling Layer}

This layer progressively reduces the spatial size to reduce the parameters and calculations of the network. Pooling layer uses MAX operations on every depth slice to reduce parameters. Filter size must be $(2 \times 2)$ with the value 2 as stride, downwards. Each sample depth is the input of 2 on the height and width which leads to discarding $73 \%$ of activations is very common in pooling layer. In this case, MAX operation take a max 4 numbers. Pooling layer also performs other functions like L2-norm pooling or average pooling.

Pooling layer does:

$>$ Accepts a volume of size

Where [w1 = H1 = D1]

$\mathrm{W} 1 \times \mathrm{H} 1 \times \mathrm{D} 1 \mathrm{~W} 1 \times \mathrm{H} 1 \times \mathrm{D} 1$

Where,

$\mathrm{W}=$ width

$\mathrm{H}=$ Height

$\mathrm{D}=$ dimensions

$>$ Requires two hyper parameters.

1. Spatial extent.

2. Stride.

$>$ Produces a volume of size.

$\mathrm{W} 2 \times \mathrm{H} 2 \times \mathrm{D} 2 \mathrm{~W} 2 \times \mathrm{H} 2 \times \mathrm{D} 2$

Where

$$
\begin{gathered}
\mathrm{H} 2=(\mathrm{H} 1-\mathrm{F}) / \mathrm{S}+1 \\
\mathrm{~W} 2=(\mathrm{W} 1-\mathrm{F}) / \mathrm{S}+1 \\
\mathrm{D} 2=\mathrm{D} 1
\end{gathered}
$$

\subsection{Normalization Layer}

This was proposed to use in CNN with intentions of implementing its inhibition schemes which are observed in a biological brain. These will decrease models training time which makes a huge impact. Each feature is normalized which makes it easy as many weights might be higher or lower/unbiased. It reduces internal covariate shift which means a change of network parameters while training which Improves training.

\subsection{Fully Connected Layer}

This takes outputs of previous layers and turns that into a single vector layer which can be an input for the next steps and backpropagation. Takes inputs from feature analysis then apply weights to predict correct labels. This gives the final probability of each label. Their activations can be computed with matrix multiplication followed with bias offset.

Layer pattern would be:

$$
\text { INPUT }->\left[[C O N V ~->\text { RELU }]^{*} \mathrm{~N}->\text { POOL? }\right]^{*} \mathrm{M}->[\mathrm{FC}->\text { RELU]*K->FC }
$$

What generally happens inside a neural network? 

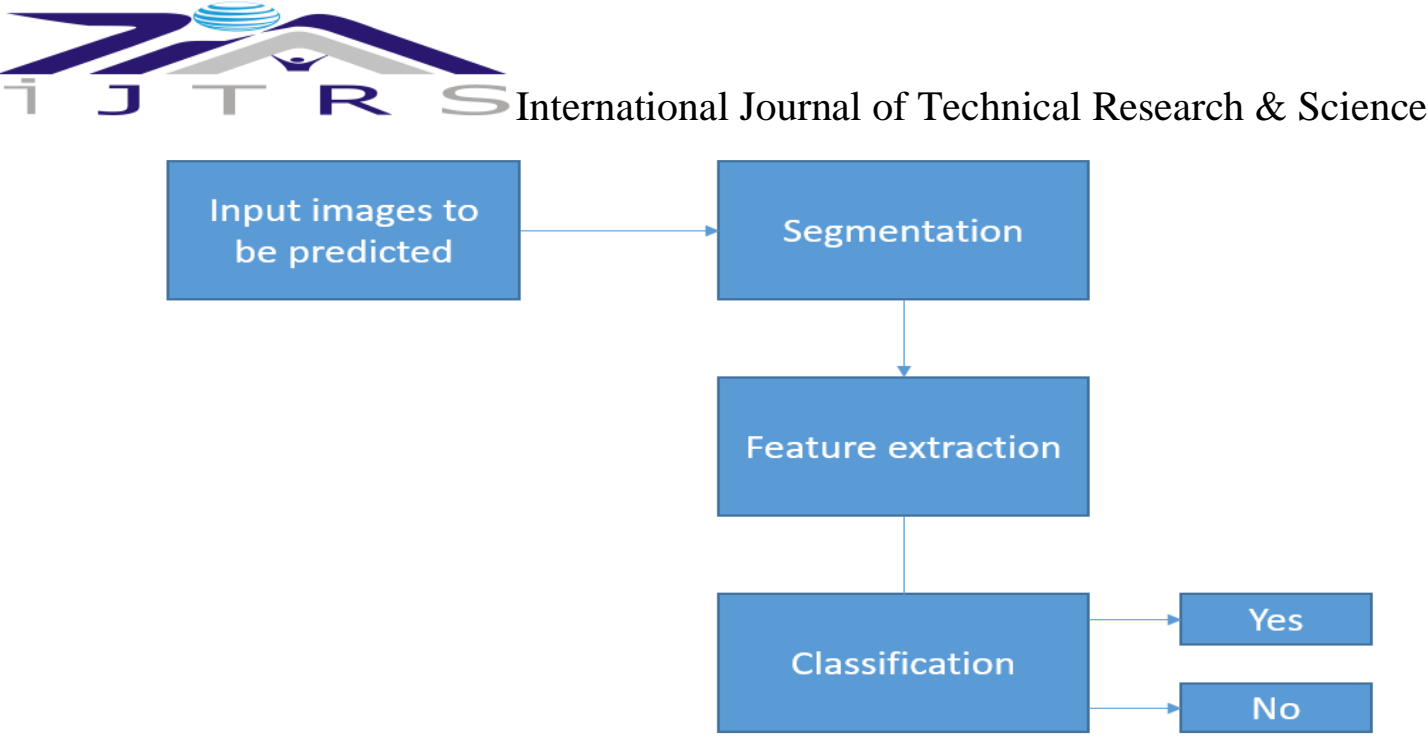

Fig. 7.7 Block diagram for predicting Skin Cancer

In the above Fig. 7.7, explanation of terms:

Skin cancer images to be predicted: These consists of the images of the skin that a user wants to detect the probability of diagnosed with what type of cancer.

\subsection{Segmentation}

This is the most crucial part of image processing, as it divides the image into visual input then into segments to analysis image i.e. identifying parts of images understanding them which object they belong to, this is the basic step for object detecting. There are types in segmentations they are Semantic segmentation, Instance segmentation. Semantic segmentation means it classifies objects into different classes and correspond them to real-world categories and then colour them to identify by the model and also known as a dense prediction as this predicts each pixel's meaning. And whereas instance segmentation means it recognizes each instance of an object from the image. This classifies every pixel.

\subsection{Feature Extraction}

This describes detailed shapes information contained in pattern and classifies those and make them easy to recognize, this helps in finding relevant information about an object from original data then it represents same information in reduced data such as dimensionality reduction.

Classification: This whole paper is related to classification hence we will learn more about it further. Classification means extracting class's information from an image as it consists of many different objects and then classifying it, this is used to create "thematic maps. There are 2 types of classifications namely supervised and unsupervised.

\section{MODEL PIPELINE}

To Build the CNN Model which was used to detect skin cancer, we go through the above-written process of the pipeline:

D Importing required packages such as Keras Packages, Libraries and TensorFlow.

$>$ Initializing the Convolutional neural networks.

$>$ Adding the Convolution Layers.

$>$ Adding the Max-Pooling layer.

$>$ Flattening those above layers.

$>$ Fully connecting above layers.

$>$ ConvNets compilation.

$>$ Model fitting to the dataset.

Let us see how these are used in the current algorithm for s=detecting skin cancer:

\subsection{Import Keras Packages and Libraries, TensorFlow}

From Keras.layers import Dense, Flatten, Convolution3D, MaxPooling2D, Convolution2D

from Keras import Sequential

from keras.preprocessing.image import ImageDataGenerator

\subsection{Initializing the $\mathrm{CNN}$}

First, we arrange all the matrices of the image into a sequence and perform an operation on that and to do that we use keyword Sequential()

From Keras. models

Model $=$ sequential () 


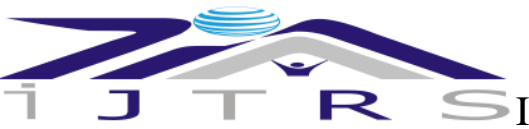

\subsection{Adding the Convolution Layer}

Then we convolute the matrices or arrays using conv2 $\mathrm{d}$ which is available in Keras. layer. model.add(convolution $2 \mathrm{~d}(64,3,3$,input_shape $=(64,64,3)$,activation='relu')

The first 64 represents a bit of the computer which generally should be 64 .

The next 3,3 are the size or dimensions.

The next 64, 64 in input shape are the matrix pixels.

The next 3 is colour channels, 3 means red blue and green channels.

$\mathrm{ReLu}$ is the activation function, which is defined as Rectifier Unit. Which activates the neurons of the CNN, it adds non-linearity to improve the efficiency of the NN it only keeps Positive values and neglects Negative values.

\subsection{Adding the Pooling Layer}

Then for the convoluted data, we apply max-pooling using keyword maxpooling2d from Keras. layers.

In max-pooling, all the training is done to the dataset: model.add(MaxPooling2D(pool_size $=(2,2))$ )

Here the attributed $(2,2)$ describe the factor by which to downscale the images.

\subsection{Adding Flattening to the Layer}

After training it, we flatten all the matrices using flatten from Keras. layers.

\subsection{Adding the Fully Connected Layer}

Now, this is the most important part of the code, the dense layer is known as a fully connected layer whose output layer which is similar to hidden layers in ANNs.

model.add(Dense(output_dim=128, activation = 'relu')

Here output dimensions are 128 because of the computer, it makes code more accurate and it should not be too small such as 32 or too big and thus 128 is accurate.

Activation function ReLu.

model.add(Dense(output_dim=1, activation = 'sigmoid')

Here in this output dimensions are 1 because we need to get one output that is either yes or no ( 0 or 1$)$

And the activation function here is sigmoid which is a type of activation function and defined as squashing function whose values would lie between 0 and 1 .

\subsection{Compiling the CNN}

Next step is to compile the CNN using compiler keyword with attributes optimizer, loss and metrics. model. compile(optimizer='adam',loss='binary_crossentropy',metrices=['accuracy'])

Adam is a stochastic gradient descent algorithm which is the fastest algorithms of all available.

The model will evaluate metrics.

Generally, metrics $=$ true prediction/total prediction.

\subsection{Fitting the CNN to my image dataset}

The model was fitted to the proposed dataset by using ImageDataGenerator function. This is used to expand dataset size artificially for creating modified images versions in the dataset and its application is for data Augmentation. It replaces original batch with new random batches of data. Then takes the new image and write it back to the model and keeps repeating this model until all the epochs are completed.

For example, If the dataset has 100 images it turns them into 1000 images.

Step 1:

Training batch is presented to it.

train_datagen $=$ ImageDataGenerator (rescale $=1 . / 255$, shear_range $=0.2$,zoom_range $=0.2$,horizontal_flip=True)

Where,

Rescale $=$ reshaping images into different pixels here it is 1./255 size as 255 is the maximum pixel value.

Shear range $=$ image will be distributed along the axis to create perception angles basically to view image from various angles as of human viewing structure.

Zoom range $=$ the value up to which the images can be zoomed and reformed

Horizontal flip $=$ rotating images into various angles.

Step 2:

Transforms every image to the batch of new random images, such as it rotates it, changes resolution..etc.

Step 3:

Now random batch is returned to model.

classifier.fit_generator(train_set,steps_per_epoch $=10000,=30$,validation_data=test_set,validation_steps $=300000$ )

Where,

A trainset is the training dataset location.

Steps per epoch are the number of batches of samples to be used in one iteration and the start of the next iteration. 


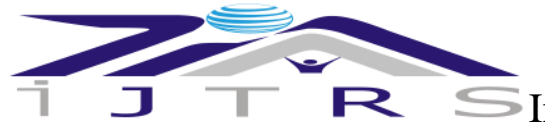

International Journal of Technical Research \& Science

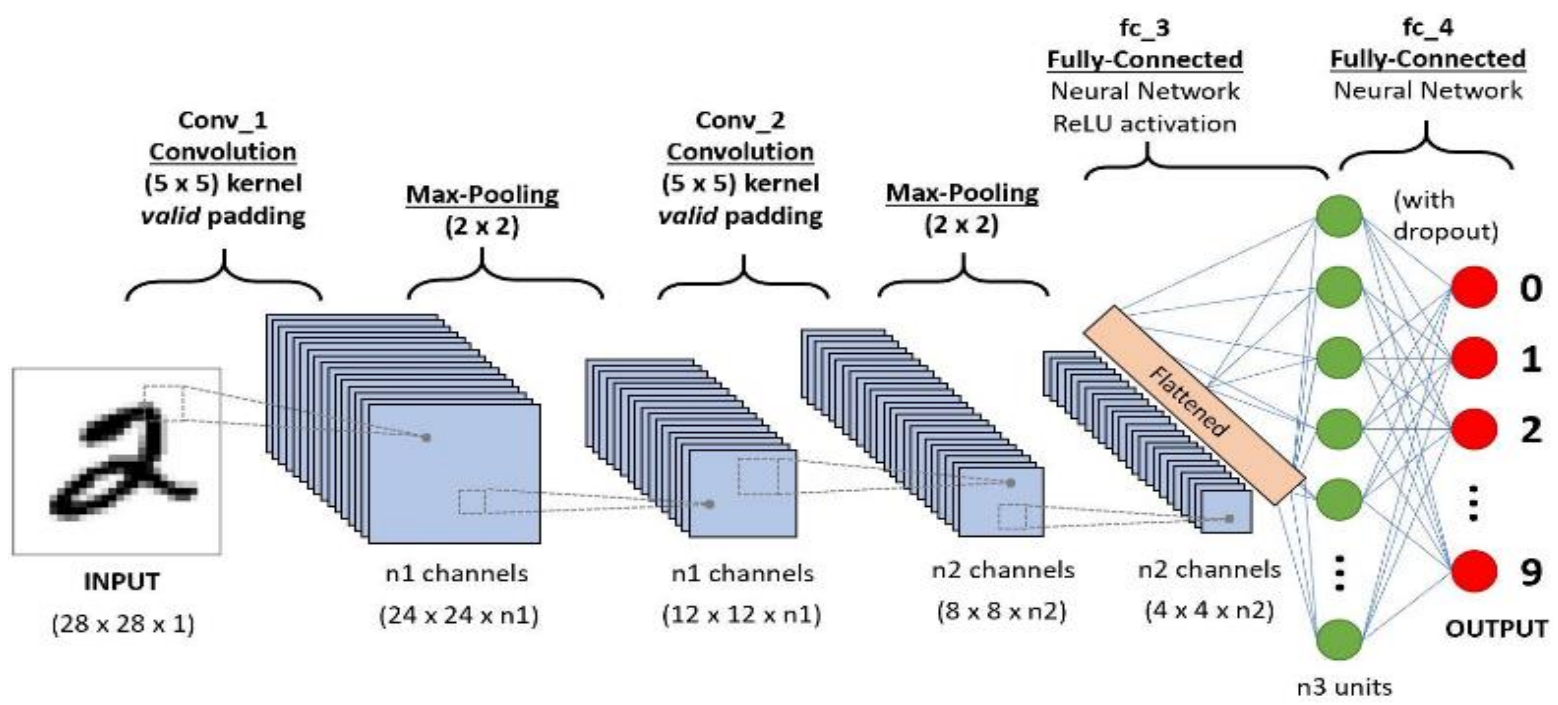

Fig. 8.1 Step by step image processing in a Neural Network

\section{RESULTS}

The present paper differentiates between 3 types of skin cancer and benign. They are Squamous cell carcinoma, Basal cell carcinoma and malignant type of cancers.

If the value is 0 then its Basal cell carcinoma, if the output value is 1 it is benign, if the output value is 2 then its melanoma and if the value of output is 3 then its Squamous cell carcinoma.

Model is shown an accuracy of $91.74 \%$ on trained data and it shows value accuracy of $87.33 \%$. Here are the screenshots that show the accuracy of the model.

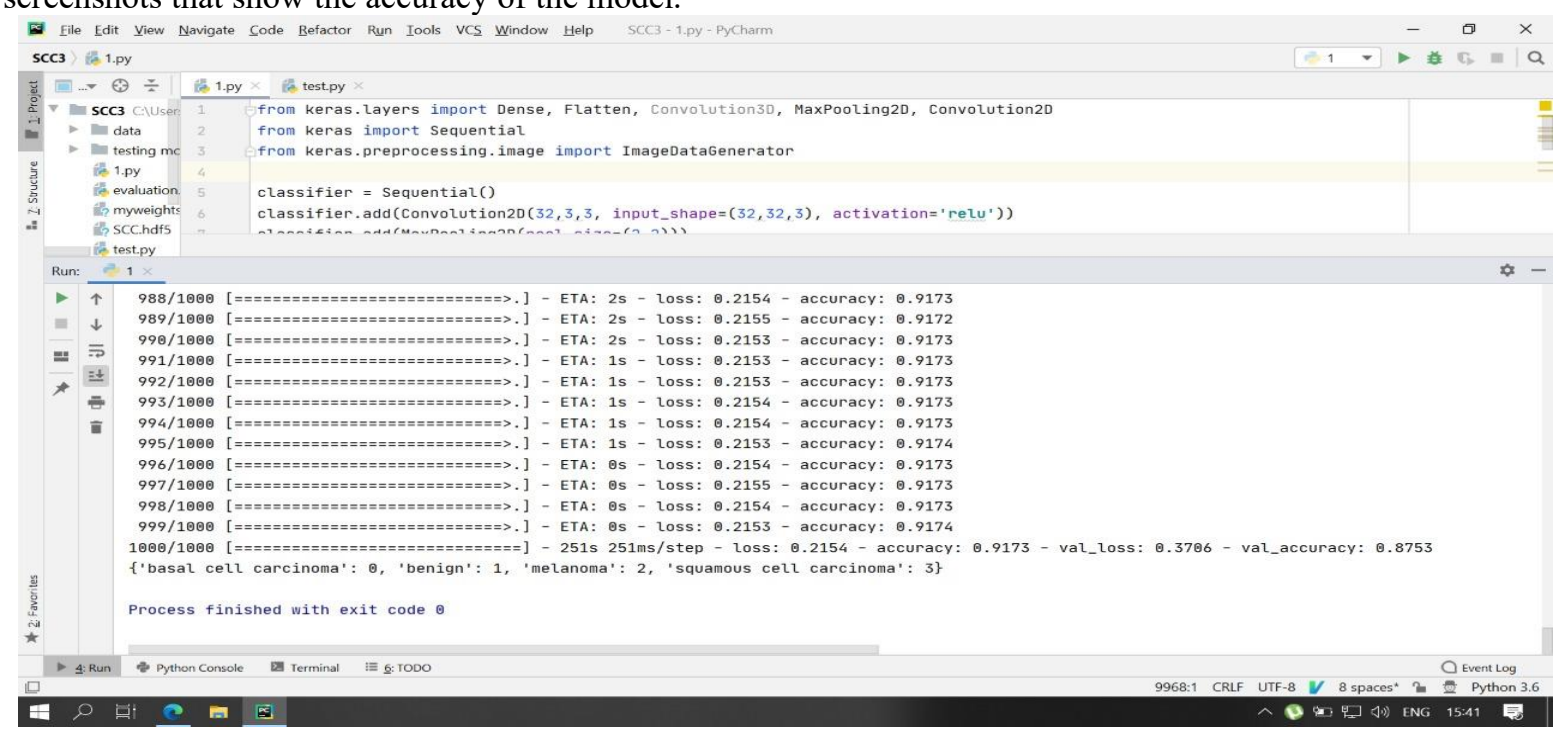

Fig. 9.1 The Output of the Model along with the Classification of types of Skin Cancer

These are not permanent values by the change in a dataset and by the amount of dataset value of accuracies change.

\section{CONCLUSION}

Skin cancer is a deadly disease which is safely diagnosed when detected early and for maintaining a healthy lifestyle. One of the accurate ways to detect skin cancer early is by using a machine learning algorithm which is Convolutional Neural Networking. This paper contains the methodology of detecting skin cancer with accuracy greater than $90 \%$ as $\mathrm{CNN}$ is very powerful and solely made for image processing. We train different types of skin cancer cells through image format and apply $\mathrm{CNN}$ over them so that it can perform well. Dataset is taken manually from different websites and in real-world through surveys. We have compared different types of algorithms used to detect skin cancer in this paper. Hence, after a comparison of different algorithms, CNN is turned out to be the best algorithm to detect skin cancer.

\section{REFERENCES}

[1] A. A. Ali and H. Al-Marzouqi, "Melanoma detection using regular convolutional neural networks," 2017 Int. Conf. Electr. Comput. Technol. Appl. ICECTA 2017, vol. 2018-Janua, pp. 1-5, 2017. 


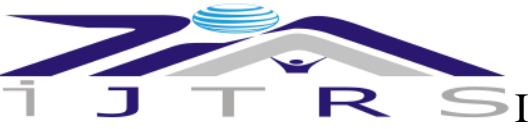

[2] P. M. Amulya and T. V. Jayakumar, “A study on melanoma skin cancer detection techniques,” Proc. Int. Conf. Intell. Sustain. Syst. ICISS 2017, no. Iciss, pp. 764-766, 2018.

[3] A. Naeem, M. S. Farooq, A. Khelifi, and A. Abid, "Malignant Melanoma Classification Using Deep Learning: Datasets, Performance Measurements, Challenges and Opportunities," IEEE Access, vol. 8, pp. 110575-110597, 2020.

[4] A. F. Agarap, "An architecture combining convolutional neural network (CNN) and support vector machine (SVM) for image classification,” arXiv Prepr. arXiv1712.03541, 2017.

[5] E. Jana, R. Subban, and S. Saraswathi, "Research on Skin Cancer Cell Detection Using Image Processing," 2017 IEEE Int. Conf. Comput. Intell. Comput. Res. ICCIC 2017, 2018.

[6] R. L. Siegel, K. D. Miller, and A. Jemal, “Cancer statistics, 2016,” CA. Cancer J. Clin., vol. 66, no. 1, pp. 7-30, 2016.

[7] M. A. Albahar, "Skin lesion classification using a convolutional neural network with novel regularizer," IEEE Access, vol. 7, pp. 38306-38313, 2019.

[8] U.-O. Dorj, K.-K. Lee, J.-Y. Choi, and M. Lee, "The skin cancer classification using deep convolutional neural network," Multimed. Tools Appl., vol. 77, no. 8, pp. 9909-9924, 2018.

[9] M. A. Kassem, K. M. Hosny, and M. M. Fouad, "Skin lesions classification into eight classes for ISIC 2019 using deep convolutional neural network and transfer learning," IEEE Access, vol. 8, pp. 114822-114832, 2020.

[10] P. Tschandl et al., "Expert-level diagnosis of non-pigmented skin cancer by combined convolutional neural networks," JAMA Dermatology, vol. 155, no. 1, pp. 58-65, 2019.

[11] C. Barata and J. S. Marques, "Deep learning for skin cancer diagnosis with hierarchical architectures," Proc. - Int. Symp. Biomed. Imaging, vol. 2019-April, no. Isbi, pp. 841-845, 2019.

[12] R. S. Sanketh, M. Madhu Bala, P. V. N. Reddy, and G. V. S. Phani Kumar, "Melanoma Disease Detection Using Convolutional Neural Networks," Proc. Int. Conf. Intell. Comput. Control Syst. ICICCS 2020, no. Iciccs, pp. 1031-1037, 2020.

[13] H. R. Mhaske and D. A. Phalke, "Melanoma skin cancer detection and classification based on supervised and unsupervised learning," 2013 Int. Conf. Circuits, Control. Commun. CCUBE 2013, pp. 1-5, 2013.

[14] F. K. Nezhadian and S. Rashidi, "Melanoma skin cancer detection using colour and new texture features," 19th CSI Int. Symp. Artif. Intell. Signal Process. AISP 2017, vol. 2018-Janua, pp. 1-5, 2018.

[15] R. Suganya, "An automated computer aided diagnosis of skin lesions detection and classification for dermoscopy images," in 2016 International Conference on Recent Trends in Information Technology (ICRTIT), 2016, pp. 1-5.

[16] H. N. Pham et al., "Lesion Segmentation and Automated Melanoma Detection using Deep Convolutional Neural Networks and XGBoost,” Proc. 2019 Int. Conf. Syst. Sci. Eng. ICSSE 2019, pp. 142-147, 2019.

[17] D. H. Murphree and C. Ngufor, "Transfer learning for melanoma detection: Participation in ISIC 2017 skin lesion classification challenge," arXiv Prepr. arXiv1703.05235, 2017.

[18] M. Ashcroft, L. Kaati, and M. Meyer, “A Step Towards Detecting Online Grooming-Identifying Adults Pretending to be Children," Proc. - 2015 Eur. Intell. Secur. Informatics Conf. EISIC 2015, pp. 98-104, 2016.

[19] E. Chabi Adjobo, A. T. Sanda Mahama, P. Gouton, and J. Tossa, "Proposition of convolutional neural network-based system for skin cancer detection,” Proc. - 15th Int. Conf. Signal Image Technol. InternetBased Syst. SISITS 2019, pp. 35-39, 2019.

[20] S. Pathan, K. G. Prabhu, and P. C. Siddalingaswamy, "Techniques and algorithms for computer-aided diagnosis of pigmented skin lesions-A review," Biomed. Signal Process. Control, vol. 39, pp. 237-262, 2018.

[21] K. Ramlakhan and Y. Shang, "A mobile automated skin lesion classification system," in 2011 IEEE 23rd International Conference on Tools with Artificial Intelligence, 2011, pp. 138-141. 